\title{
Geflüchtete Yezidinnen aus dem Irak: Leben mit der kollektiven und individuellen Verfolgungsvergangenheit
}

\author{
Christian Jorgow
}

\section{Einleitung}

Im folgenden Beitrag beschäftige ich mich mit den Lebensgeschichten und gegenwärtigen Zukunftsperspektiven von yezidischen Frauen aus dem Irak, die sich angesichts von aussichtslosen oder gewaltsamen Kontexten und Situationen zu einer Flucht aus dem Irak entschieden haben. Hierbei gehe ich den Fragen nach, welche Prozesse zu einer Flucht beigetragen haben, was sich im Leben der Geflüchteten durch die Flucht verändert hat und wie sich deren gegenwärtiges Leben gestaltet. Im Fokus steht die ethno-religiöse Minderheit der Yezid*innen. Viele Angehörige dieser Gruppierung haben seit Ende der 2000er Jahre die Flucht aus dem Irak ergriffen, um sich selbst bzw. der eigenen Familie eine sichere Zukunftsperspektive zu ermöglichen.

Im Kontext meiner Masterarbeit (Jorgow 2019) führte und analysierte ich Interviews ${ }^{1}$ mit zwei geflüchteten Yezidinnen - Ebru Hamid und Arwa Erkis ${ }^{2}$ - aus dem

\footnotetext{
${ }^{1}$ Bei den Interviews handelt es sich um biographisch-narrative Interviews (vgl. Rosenthal 2015; Schütze 1983).

${ }^{2}$ Im Rahmen des Schutzes der Interviewten wurden alle Daten, die einen Rückschluss auf ihre Person ermöglichen, unkenntlich gemacht, verändert oder anonymisiert (u.a. Name, Wohnort).
}

Jorgow, C. (2020): Geflüchtete Yezidinnen aus dem Irak. Leben mit der kollektiven und individuellen Verfolgungsvergangenheit. In: Babl, E./Becker, J. (eds.): Global Processes of Flight and Migration. The Explanatory Power of Case Studies / Globale Flucht- und Migrationsprozesse. Die Erklärungskraft von Fallstudien. Göttingen: Göttingen University Press, 255-273. https://doi.org/10.17875/gup2020-1324 
Irak. Arwa flüchtete 2009 und Ebru 2015 nach Deutschland. In diesem Beitrag werden die Interviewten und ihre Lebensgeschichten vorgestellt. Dabei werden die biographischen Verläufe in gesellschaftliche und kollektivgeschichtliche Rahmenbedingungen und Entwicklungen eingebettet, um sich den Perspektiven der Interviewten verstehend anzunähern. Die größtenteils im Nordirak lebende Gruppierung der Yezid*innen, deren Mitglieder sich überwiegend der kurdischen Bevölkerung zuschreiben (siehe auch den Beitrag von Sevil Çakır-Kılınçoğlu in diesem Band), ist eine in Deutschland bislang kaum wahrgenommene ethno-religiöse Minderheit, welche erst mit dem Einmarsch des IS (sog. Islamischer Staat) bzw. Daesh in den Nordirak im Juli 2014 vereinzelt mediale Aufmerksamkeit erhalten hat. Dies ist erstaunlich, wenn man beachtet, dass es mit ca. 150.000 Menschen in Deutschland mittlerweile die weltweit größte yezidische Diaspora gibt (vgl. Tagay/Ortac 2016). Auch wenn sich in der Religions-, Sozial- und Migrationsforschung wissenschaftliche Abhandlungen mit Yezid*innen und dem Yezidentum auseinandersetzen (vgl. Acikyildiz 2010; Kartal 2016; Oehring 2017; Savucu 2016; Tagay/Ortac 2016), fehlt bislang eine stärker an die Perspektive der Akteur*innen und deren Lebens- und Migrationsverläufe gebundene Betrachtung. Zu einer solchen möchte ich mit diesem Kapitel beitragen. Beide Interviewte wuchsen in geschlossenen yezidischen Communities im Nordirak auf. Sie waren Teil von Großfamilien, bis sie sich zur Flucht aus einer prekären bzw. lebensbedrohlichen Situation entschieden haben oder gezwungen sahen. Beide Frauen haben gemeinsam, dass die Verfolgungsgefahr, die mit der Zugehörigkeit zur yezidischen Community verbunden ist, ein Leben in Sicherheit im Irak verunmöglichte. Zum einen Arwa, die 2009 mit ihrer Familie über das Mittelmeer in Richtung Deutschland flüchtete, um einer perspektivlosen und prekären Situation im Irak zu entgehen. Zum anderen Ebru, die im Jahr 2015 flüchtete, nachdem sie im August 2014 den Genozid an den Yezid*innen im Nordirak überlebt hatte. Die Interviewten mussten auf ihrer Flucht verschiedene Hürden und Grenzen überwinden, Schlepper bezahlen und um die Anerkennung ihrer Schutzbedürftigkeit kämpfen. Ihr Ziel war es, einen sicheren Ort in Deutschland zu erreichen. Dies bedeutete unter anderem, die Familie den Gefahren einer Mittelmeerüberquerung in einem Schlauchboot auszusetzen. Es bedeutete auch, die eigene Zugehörigkeit zu verschweigen, um Verfolgung und Diskriminierung während der Flucht aus dem Weg zu gehen. In Deutschland angekommen, versuchten beide eine langfristige Bleibeperspektive zu ermöglichen, wobei sie auf die Präsentation und Anerkennung ihrer individuellen Verfolgungsgeschichte angewiesen waren. Um eine dauerhafte Aufenthaltserlaubnis zu erhalten, mussten Ebru und Arwa zudem die deutsche Sprache erlernen, sich stärker am öffentlichen Leben beteiligen und sich mit dem behördlichen Anerkennungsverfahren auseinandersetzen. Die Bedingungen für eine Aufenthaltserlaubnis in Deutschland, die Auseinandersetzung mit dem Fremden und Neuen der Ankunftsgesellschaft sowie die Konfrontation mit verschiedensten Hürden und Entscheidungssituationen war für beide Yezidinnen zudem mit einem gewissen Emanzipationsprozess verbunden. 
Für viele Yezid*innen erscheint die Lage im Irak weiterhin als prekär, da dort eine sichere und friedliche Lebensgrundlage oftmals nicht gegeben ist. Die systematische Zerstörung von Dörfern, Städten und Ackerflächen sowie das Verminen dieser Landflächen durch den Daesh machen ein Leben in diesen Gebieten weiterhin schwierig bis unmöglich. Nur langsam kommt es zum Wiederaufbau von Infrastrukturen und zu der Räumung der unzähligen Minen. Zwar wurde der Daesh aus diesen Gebieten größtenteils vertrieben oder zurückgedrängt, die Angst vor Verfolgung dürfte für die Zivilbevölkerung jedoch fortbestehen, da die irakische Regierung weiterhin von über 20 kleineren Daesh-Zellen spricht, welche im Nordirak existieren (vgl. Pro Asyl 2019).

Gleichzeitig wurde im Juli 2019 durch das Oberverwaltungsgericht in Lüneburg ein Urteil erlassen, das den Irak für Yezid*innen als sicheres Land deklariert, womit deren Abschiebung eine legale Grundlage erhalten hat (Schirrmeister 2019). Eine Rückkehr ist für Ebru, die einen Großteil ihrer Familie durch den Genozid des Daesh an den Yezid*innen im Nordirak im August 2014 verloren hat, sowie für Arwa, die viele Gefahren auf sich genommen hat, um ihrer Familie eine lebenswerte Zukunft bieten zu können, weder sicher noch vorstellbar. Dies trifft auch auf viele weitere in Deutschland lebende Yezid*innen zu, die den Irak in den letzten Jahren verlassen haben. Die Präsenz von mehr als 100.000 Yezid*innen in Flüchtlingslagern und Städten auf dem Gebiet der Autonomen Region Kurdistan, wohin sie im Kontext des Einmarsches des Daesh im Jahr 2014 geflüchtet waren und von wo sie bis heute nicht zurückkehren können oder wollen, deutet darauf hin, dass die Lage für Yezid*innen im Nordirak nicht sicher ist (Heine 2019).

Für ein besseres Verständnis der Situation der Yezid*innen aus dem Irak ist ein Blick auf deren Diskriminierung und Verfolgung in Geschichte und Gegenwart sowie auf deren Geschlossenheit als soziale Gruppe von enormer Bedeutung. Durch die Analyse der biographischen Verläufe von yezidischen Frauen, die aus dem Irak geflohen sind, wird deutlich, dass biographische Faktoren und Erfahrungen ebenso entscheidend für eine erfolgreiche Fluchtmigration sind wie gleichzeitig ökonomisches Kapitel und soziale Netzwerke nötig sind, damit ein Verlassen des Herkunftskontextes überhaupt möglich wird. Die Verläufe zeigen zudem auf, dass Flüchtende sich mit einer Vielzahl verschiedener Grenzregime und -politiken konfrontiert sehen, durch die oftmals willkürlich definiert wird, welche Menschen ein Land betreten dürfen und welche nicht. Dadurch wird unter anderem bestimmt, welche Fluchtrouten für bestimmte Gruppierungen offen und welche geschlossen sind, und die Entscheidung der Migrant*innen beeinflusst, ob die Flucht über Land möglich ist oder die Gefahren einer Mittelmeerüberquerung riskiert werden. Während ihrer Flucht sind die hier Interviewten eine Vielzahl von Gefahren eingegangen, um einen sicheren Ankunftskontext zu erreichen. Sie verdeckten ihre Zugehörigkeit, um Grenzen zu überwinden, oder kämpften für die Anerkennung ihrer Schutzbedürftigkeit. Dabei war es für diese Fluchtverläufe typisch, dass sich Phasen abwechselten, in denen die Geflüchteten passiv und handlungsohnmächtig über sich entscheiden lassen oder Situationen gar erleiden mussten, während sie in anderen Phasen eine 
gewisse Handlungsmacht besaßen oder sich diese erkämpften, um die Sicherheit der eigenen Person oder der Familie zu sichern (vgl. Rosenthal/Hofmann in diesem Band). Ein weiteres Ergebnis der Fallanalysen ist, dass die Flucht und das Leben in einer Ankunftsgesellschaft einen wichtigen Einfluss auf die Entwicklung der eigenen Zugehörigkeitskonstruktionen ${ }^{3}$ hat, wobei biographische Erfahrungen und Erlebnisse, die vor der Flucht gemacht wurden, ebenso entscheidend für den Umgang mit der eigenen Zugehörigkeit sind. Dies bedeutet, dass die Konstruktion von Zugehörigkeit als lebenslanger Prozess zu verstehen ist, welcher eben nicht beliebig ist, sondern sich als Zusammenspiel verschiedenster (aktueller) Krisenerfahrungen und den tradierten sowie erlernten Handlungs- und Denkmustern einer sozialen Gruppe erklären lässt. Besonders herauszustellen ist hierbei das Erleben extrem traumatischer Erfahrungen im Rahmen von ethnischer Verfolgung, die den Wandel von Zugehörigkeitskonstruktionen zusätzlich verstärken. Die unterschiedlichen Verläufe von Zugehörigkeitskonstruktionen, wie ich sie bei Arwa und Ebru aufzeigen werde, machen deutlich, dass eine simplifizierende Sicht auf Fluchtursachen übersieht, welche lebensgeschichtlichen Erlebnisse und Verläufe relevant sind, damit Menschen aus prekären und lebensbedrohlichen Situationen flüchten können und ein Ankommen in einer Ankunftsgesellschaft überhaupt möglich ist. Ich gehe darüber hinaus davon aus, dass sich die empirischen Ergebnisse meiner Arbeit bezüglich der Flucht und des Ankommens nicht nur auf yezidische Menschen aus dem Irak beziehen lassen, sondern dass sich deren Charakteristik auch bei anderen Menschen und Gruppierungen finden lässt, welche aus einem aussichtslosen und/oder gewaltvollen Kontext flüchten. Zudem lässt sich nicht nur für die Gruppierung der Yezid*innen beobachten, dass Länder, die vormals als ,unsicher" galten und daher Abschiebungen dorthin nicht erlaubt waren, zunehmend als „sicher" bezeichnet werden. Somit droht einer Vielzahl von verfolgten und geflüchteten Menschen eine Abschiebung in ein Land, in welches sie nicht mehr zurückwollen, in welchem es keine Zukunftsperspektiven für sie gibt und in welchem eine Verfolgung und Kontinuität von Diskriminierung weiterhin nicht ausbleibt. ${ }^{4}$ Im Gegensatz zu den in diesem Beitrag vorgestellten Fällen, die durch ihren Aufenthaltstitel eine langfristige Bleibeperspektive in Deutschland gesichert haben, sieht sich eine Vielzahl yezidischer Geflüchteter durch die neusten rechtlichen Entwicklungen in der Gefahr, in den Irak abgeschoben zu werden, welchen sie - unter Inkaufnahme der Gefahren einer Flucht - verlassen haben. Bevor ich die untersuchten Fälle von Ebru und Arwa vorstelle, gebe ich zunächst einen Einblick in das Yezidentum und die Situation der Yezid*innen im Nordirak.

\footnotetext{
${ }^{3}$ Zum Wandel und zu der Bedeutung von Zugehörigkeitskonstruktionen bei ethnisch verfolgten und/oder flüchtenden Menschen siehe u.a. Rosenthal 2004 und Rosenthal/Bahl/Worm 2017.

${ }^{4}$ Eine ganz ähnliche Abschiebepolitik durch die deutsche Bundesregierung lässt sich bereits anhand der Abschiebungen von afghanischen Geflüchteten, welche vor allem seit 2015 nach Deutschland flüchteten, aufzeigen (vgl. Sökefeld 2019).
} 


\section{Zum Yezidentum und zur Verfolgungsgeschichte der Yezid*innen}

Die Yezid*innen sind eine religiöse Minderheit, deren Mitglieder sich ethnisch selbst größtenteils der kurdischen Bevölkerung zuschreiben und überwiegend im Nordirak, in den Provinzen Dohuk und Mosul sowie in den Regionen Sindschar und Sheikan, leben (vgl. Tagay 2018). Auch wenn es bis heute keine amtlich erhobenen Statistiken gibt, so belaufen sich Schätzungen verschiedener Autor*innen und Organisationen auf 800.000 bis 1.000 .000 Yezid*innen weltweit, wobei ein Großteil davon (ca. 600.000-700.000) im Irak lebt bzw. gelebt hat. Yezid*innen leben darüber hinaus in der Türkei, in Syrien und im Iran. Teile der Diaspora in Armenien, Georgien und Russland sowie einige Yezid*innen aus dem Sindscharbezirk im Irak verstehen sich nicht als Kurd*innen, sondern als eigenständige ethno-religiöse Gruppe. Dass über das Yezidentum bis in das 20. Jahrhundert kaum etwas bekannt war, liegt dabei auch an der Verfolgungsgeschichte, die zur Folge hat, dass religiöse Praktiken und Zugehörigkeiten geheim gehalten wurden (vgl. Tagay/Ortac 2016). Yezid*innen aus dem Irak sprechen größtenteils den nordkurdischen Dialekt Kurmandschi, womit sie sich von der Arabisch sprechenden Mehrheit des Landes unterscheiden. Eine Besonderheit der yezidischen Religion ist, dass sie nicht missionarisch sondern endogam ist. Man kann also nicht zum Yezidentum konvertieren, sondern muss als Kind yezidischer Eltern geboren werden, womit sich die Gruppierung als relativ exklusive Gemeinschaft versteht. Die Überlieferung der Religion findet fast ausschließlich mündlich statt, weshalb der Erhalt und die Weitergabe der Religion durch Geistliche einen wichtigen Stellenwert besitzen. Durch ihre besondere Glaubenspraxis und Abgrenzung zu anderen Schriftreligionen wurden und werden Yezid*innen immer wieder als „Ungläubige“ betitelt, was u.a. die Grundlage für ihre jahrhundertelange Verfolgung und Unterdrückung lieferte (vgl. ebd.). In ihrer Geschichte erlebten Yezid*innen eine Vielzahl von Pogromen, welche vor allem zu Zeiten des Osmanischen Reiches (ca. 1300-1920) stattfanden. Durch Tötungen, Entführungen und Zwangsislamisierungen ist die Zahl der Yezid*innen drastisch gesunken (vgl. Dinnayi 2004). Phasen der Unterdrückung und Verfolgung wechselten sich mit Phasen der Tolerierung und Zusammenarbeit mit den - in unmittelbarer Nachbarschaft lebenden - nicht-yezidischen Kurden und der arabischen Bevölkerung ab. So gab es z.B. kurz nach der Gründung des Osmanischen Reiches Bestrebungen von Kurd*innen und Yezid*innen zur Bildung eines eigenen Emirates, welches jedoch durch das Osmanische Reich niedergeschlagen wurde. Vor allem ab dem 16. Jahrhundert nahm der politische Einfluss yezidischer Stämme zunehmend ab, während die eigene Bevölkerung immer öfter Opfer von Verfolgung und Ausgrenzungspraktiken wurde (vgl. Tagay/Ortac 2016).

Der Ursprung der yezidischen Religion wird noch vor das Jahr 2000 v.u.Z. datiert. Ihre Wurzeln finden sich in verschiedenen Praktiken alt-iranischer Religionen. Durch die fehlenden schriftlichen Überlieferungen ist sie ein historisches Produkt oraler Tradierungen. Da es zudem keinen offiziellen Religionsunterricht gibt und die 
Tradierung des religiösen Wissens der Familie sowie den Geistlichen eines Dorfes zukommt, gibt es durchaus regionale Unterschiede in der Religionspraxis. Trotzdem lassen sich einige Grundannahmen zusammenfassen. So ist das Yezidentum eine monotheistische Religion, welche gewisse Normen und Riten vorgibt. Es gibt ein Kastenwesen (drei Hauptkasten), in welche Yezid*innen geboren werden und nur innerhalb derer sie heiraten dürfen, womit gewisse gesellschaftliche Strukturen aufrechterhalten werden. Eine Heirat außerhalb der Kaste sowie außerhalb der Gemeinschaft führt zum Ausschluss aus der Community (vgl. Schulz 2009).

Wie sich auch in den in diesem Artikel diskutierten Lebensgeschichten zeigt, hat sich die Stellung der Frau in yezidischen Familien über die letzten Jahrzehnte verbessert. Trotzdem ist das Familienbild von patriarchalen Strukturen geprägt, wodurch vielen Frauen der Zugang zu Bildung und/oder Erwerbsarbeit weiterhin versperrt bleibt. Obwohl die Heirat seit 1929 auf Basis einer selbstbestimmten Partnerwahl beruhen soll, so hatte es der religiöse Rat der Yezid*innen beschlossen, kommt es im Nordirak auch heute noch zu Zwangsehen (vgl. Tagay/Ortac 2016). Als Angehörige einer religiösen Minderheit sowie durch das Vorhandensein von patriarchalen Strukturen laufen yezidische Frauen daher Gefahr, doppelt diskriminiert zu werden. Im Kontext der irakischen Gesetzgebung sind Frauen Männern zudem nicht gleichgestellt, womit eine Strafverfolgung von Tätern, welche sexuelle Gewalt gegen Frauen ausüben, diese entführen oder sogar töten, oftmals ausbleibt. Für yezidische Frauen, die in der Regel kein Kopftuch tragen, bedeutet dies für das Leben in konservativ-muslimischen Regionen, dass ihre Freiheit, sich im Alltag außerhalb der Community zu bewegen, stark eingeschränkt ist. Um trotzdem am öffentlichen Leben teilnehmen zu können, sehen sich viele Frauen daher genötigt, ihre Zugehörigkeit zu verschleiern und sich zum eigenen Schutz konservativen islamischen Kleidungskonventionen unterzuordnen. Falls es zu einer Entführung und/oder einem sexuellen Übergriff kommt, verliert die Betroffene aufgrund des Gebotes der sexuellen Unantastbarkeit durch Nicht-Yeziden ihre Zugehörigkeit zur yezidischen Gemeinschaft (vgl. Schulz 2009). Bis in die 1950er Jahre setzten die geistlichen Oberhäupter ein Lese- und Schreibeverbot außerhalb der geistlichen Kasten durch, das bis heute Nachwirkungen hat (vgl. Savucu 2016). In den 1960er Jahren entwickelte sich jedoch ein gesellschaftliches Streben nach Bildung, welches den Folgegenerationen eine bessere Zukunftsperspektive bieten sollte. Anders als zuvor unterstützten die geistlichen Oberhäupter diese Entwicklung nun sogar, da sie im Zugang zu Bildung eine existenzsichernde Perspektive für die Yezid*innen und das Yezidentum erkannten. Dies hatte zwar zur Folge, dass die Analphabet*innenrate seither stark gesunken ist und immer mehr yezidische Männer Zugang zum Bildungssystem erhielten. Den meisten Frauen blieb die Möglichkeit, die Schule zu besuchen, allerdings weiterhin verwehrt (vgl. Kartal 2016).

Zeitgleich kam es immer wieder zu größeren Fluchtmigrationen von Yezid*innen aus dem Irak ins nahe und ferne Ausland, welche insbesondere durch die „Arabisierungskampagne" der Baath-Partei ab 1968 forciert wurden. Viele Yezid*innen wurden zudem aus ihren Herkunftsgebieten vertrieben und in Modelldörfer zwangs- 
umgesiedelt. Die Verfolgung sowie die Diffamierung als Ungläubige trugen zu Entführungen, Tötungen und zur Zerstörung ganzer Dörfer bei. Vor der ersten großen Auswanderungsbewegung in den 1990er Jahren lebten im Nordirak etwa 800.000 Yezid*innen (vgl. Ibrahim 2011), die weltweit größte Community. Im Rahmen des Einmarschs der USA in den Irak im März 2003, mit dem der Dritte Golfkrieg begann, wurde Saddam Hussein zwar abgesetzt, die jahrelange Besatzungszeit eröffnete aber zugleich radikal-islamistischen Netzwerken wie al-Qaida die Möglichkeit, sich im Irak auszubreiten (vgl. Buchta 2016). Im Jahr 2007 kam es zu mehreren Selbstmordattentaten im Sindschardistrikt, die mit al-Qaida in Verbindung gebracht wurden, bei denen über 790 Zivilisten getötet und weitere 1.500 verletzt wurden, die meisten davon Yezid*innen. Seither stellen die Milizen der Autonomen Region Kurdistan im Nordirak Polizeikräfte zur Verfügung, um die yezidische Minderheit sowie weitere ethnische Gruppierungen im Nordirak vor Verfolgung zu schützen. Darüber hinaus versuchte die kurdische Unabhängigkeitsbewegung, auch gesellschaftspolitische Prozesse wie z.B. die Stärkung der Frauenrechte innerhalb der ethnischen Gruppierungen voranzutreiben. Mit dem Aufkommen des Daesh stieg die Verfolgungsgefahr für Yezid*innen im Irak weiter an und erreichte im Sommer 2014 einen grausamen Höhepunkt. Als der Daesh im Juni 2014 nach Mosul und im August 2014 in das Sindschargebiet einmarschierte, flüchteten viele (nicht nur) yezidische Bewohner*innen in sicherere kurdische Gebiete oder in das Sindschargebirge und warteten dort zumeist in Notunterkünften und ohne jegliche Grundversorgung auf Hilfe. Die im Sindschargebiet zurückgebliebene Bevölkerung wurde zeitgleich Opfer eines durch den Daesh ausgeführten Genozides, bei dem über 5.000 Yezid*innen ermordet und mehr als 6.000 Frauen und Kinder entführt, versklavt und/oder verkauft wurden.

Erst durch die Militäroffensive einer Anti-IS-Koalition konnte der Daesh im September 2014 langsam zurückgedrängt werden. Wegen der fehlenden Sicherheit lebten noch im Jahr 2016 über 300.000 Yezid*innen in Flüchtlingslagern der kurdischen Regionalregierung. Doch auch die Bedingungen innerhalb der Flüchtlingslager sind katastrophal, da es oftmals kein sauberes Trinkwasser gibt, die medizinische Versorgung kaum ausreicht, Stromversorgung nur partiell vorhanden ist und die teils stark traumatisierten Opfer kaum psychologische und psychotherapeutische Unterstützung erhalten (vgl. Tagay/Ortac 2016). Aufgrund der fehlenden Bleibeperspektive entschieden sich viele Yezid*innen, das Land zu verlassen, viele von ihnen flüchteten nach Deutschland, wie sich an den Asylanträgen von 2015 und 2016 ablesen lässt. 2015 stellten 15.000 Yezid*innen aus dem Irak in Deutschland einen Erstantrag auf Asyl, 2016 waren es sogar über 37.000 (Bundesamt für Migration 2017).

Zwar wurde der Daesh im Irak seit Mitte 2018 offiziell als besiegt erklärt, da kaum noch größere Städte besetzt waren. Es ist jedoch davon auszugehen, dass sich übriggebliebene Kämpfer in abgelegene Gebiete des Landes zurückgezogen haben oder untergetaucht sind und daher auch weiterhin eine Gefahr für die Zivilbevölkerung darstellen (vgl. Kizilhan 2019). Nach diesem Einblick in die historische und 
politische Situation der Yezid*innen im Irak stelle ich im Folgenden die Fälle der interviewten Yezidinnen Ebru und Arwa vor.

\section{Ebru, die Neustarterin, die alles verloren hat}

Ebru (geboren 1995) hatte bereits zwei Jahre in Deutschland gelebt, als ich sie im Sommer 2018 in einer hessischen Großstadt zu ihrer Lebensgeschichte interviewte. Sie ist im August 2014 aus dem Nordirak vor dem IS-Genozid in der Sindscharregion geflüchtet. Dieser Fall repräsentiert einen lebensgeschichtlichen Verlauf, der von einem extrem traumatischen und gewaltvollen Bruch geprägt wurde. Die kollektive und individuelle Verfolgungsgeschichte bedingt auch Ebrus Gegenwart sowie ihren Blick auf die Zukunft. Die Erkenntnisse, welche sich aus der erzwungenen Flucht aus dem gewaltvollen und verlustreichen Kontext bei Ebru herausarbeiten ließen, lassen sich verlaufstypisch auch in vielen weiteren Fluchterfahrungen von Menschen wiederfinden, welche die Flucht aus einem ähnlichen Kontext ergriffen haben. Über eine Dolmetscherin wurde mir der Kontakt zu Ebru ermöglicht. Da Ebru zum Zeitpunkt des biographischen Interviews hauptsächlich Kurmandschi sprach, wurde dieses von mir auf Deutsch geführt und von der Dolmetscherin übersetzt. Ich traf mich zu drei Interviewsessions mit Ebru. Bereits zu Beginn des ersten Treffens wurde deutlich, dass Ebru ihre Erzählungen im Rahmen ihrer Verluste und der Verfolgung ihrer eigenen Person, aber auch der yezidischen Bevölkerung präsentieren muss, da diese Erlebnisse ihren Blick auf ihre Vergangenheit stark prägen. Dies wird auch anhand ihrer Eingangserzählung deutlich:

Ich heiße Ebru, ich komme aus Sindschar, Irak, geboren in Kocho. Ich habe sechs Geschwister und drei Brüder, Mutter und Vater gehabt. Ich bin 22 Jahre alt. Anfang August 2014 hat die Flucht vor dem Daesh angefangen. Ich, mein Mann und meine Tochter sind abgehauen, wir sind Richtung Kurdistan. In der Fluchtzeit sind mein Mann und meine Tochter durch den Daesh gestorben.

In den beiden weiteren Gesprächen thematisierte Ebru vor allem ihre persönliche sowie die kollektive Verfolgungsgeschichte der Yezid*innen, wobei sie jedoch ein wenig stärker auf Erzählungen ihrer Lebensgeschichte vor der Flucht eingehen konnte, was ich als Vertrauensgewinn durch mein aktives Zuhören und mein Interesse an ihrer gesamten Lebensgeschichte wahrgenommen habe. Dass Ebru bereits so offen und intensiv über ihre verlustreiche Geschichte erzählen kann, ist sicherlich auch den Psychotherapien zu verdanken, die Ebru in Deutschland wahrnehmen konnte und die sie dabei unterstützen, ihre Traumata zu bearbeiten.

Die Antworten auf Fragen zu ihrer Lebensgeschichte vor dem Zeitpunkt ihrer Verluste fielen insgesamt aber trotzdem spärlich aus. Es zeigte sich, dass Ebru zum einen mit einer starken Überlebensschuld kämpft, da sie den Angriff durch den Daesh überlebte, während viele andere ermordet wurden. Zum anderen ist Ebru durch ihre yezidische Zugehörigkeit geprägt. Sie sieht durch die Darstellung der Verfolgungsgeschichte eine Möglichkeit, sich für die Anerkennung der Schutzbedürftig- 
keit auszusprechen. Dies kann dabei auch als Ermächtigungsstrategie verstanden werden, welche Ebru hilft, der Situation der Handlungsohnmacht zu entkommen. Im Folgenden soll dies anhand der Darstellung von Ebrus Fallgeschichte aufgezeigt werden.

Ebru wurde im Jahr 1995 im ländlichen Kocho im Nordirak in eine Großfamilie geboren. Zum Zeitpunkt von Ebrus Geburt litt die irakische Bevölkerung noch unter den Folgen des Zweiten Golfkrieges (1990-1991) und dem anhaltenden UNEmbargo (seit 1990). Der Irak war wirtschaftlich isoliert und politisch destabilisiert. Durch das Aufwachsen in einer geschlossenen yezidischen Dorfgemeinschaft sowie durch die Subsistenzwirtschaft und Selbständigkeit der Eltern blieb Ebrus Familie von dieser gesamtgesellschaftlichen Situation jedoch eher verschont. Ebrus Kindheit und Jugend gestalteten sich wegen der Verfolgungsgefahr für Yezid*innen und deren Diskriminierung sehr behütet im geschützten Raum des Familienhauses und des Heimatdorfes im Nordirak. Im Vergleich zu vielen anderen Yezidinnen in ihrem Alter erfuhr Ebru - anders als Arwa (s.u.) - eine basale Bildung und lernte lesen und schreiben. Gleichzeitig begann 2003 der Dritte Golfkrieg, der mit der anschließenden Besetzung des Iraks durch eine von den USA angeführte militärische Koalition weitere Destabilisierungen und Unruhen für die irakische Bevölkerung bedeutete. In den Folgejahren nahmen die Diskriminierungen, Einschüchterungen, Zwangskonversionen und gewalttätigen Angriffe gegenüber den Angehörigen von Minderheiten zu. Zu einer steigenden Gefahr wurden radikale und extremistisch-religiöse Gruppierungen, die während der Besatzung durch die USA an Relevanz gewannen. Ebru war 12 Jahre alt, als es im Jahr 2007 zu einer Reihe von Selbstmordattentaten der alQaida im Sindschargebiet kam, bei denen fast 800 Menschen starben.

Im Jahr 2012, Ebru war 17 Jahre alt, entschieden sich Ebrus Eltern, in Kocho ein Haus zu bauen, womit sie sich auch langfristig im Irak verortet sahen. Im gleichen Jahr lernte Ebru in Sindschar-Stadt - der größten yezidischen Stadt im Sindschargebiet - ihren Freund kennen und entschied sich gegen den Willen ihrer Eltern, diesen noch im selben Jahr zu heiraten. Damit positionierte sie sich gegen deren traditionelles Verständnis, dass die Ehepartner durch die Eltern der Kinder ausgewählt werden. Ebru verließ das Haus ihrer Familie und lebte bei ihrem Ehemann in Sindschar-Stadt. Im Frühjahr 2014 bekam sie ihr erstes Kind. Am 3. August 2014 marschierte der Daesh in das Sindschargebiet ein. Während Ebru und ihre Kleinfamilie beschlossen, die Stadt zu verlassen, wurde ein Großteil von Ebrus Herkunftsfamilie durch den Daesh umzingelt und Opfer des Massakers von Kocho. Ebru verlor alle ihre Brüder und ihre Eltern. Ebrus Schwestern sowie alle überlebenden Bewohner*innen des Dorfes wurden verschleppt, vergewaltigt, verkauft oder hingerichtet.

Gleichzeitig misslang Ebrus Flucht mit ihrer Kleinfamilie mit einem LKW. Sie wurden vom Daesh angegriffen, wobei ihr Ehemann und ihr sechs Monate altes Kind starben. Ebru überlebte, erlitt jedoch schwere Verbrennungen und verlor ihren rechten Unterarm. Sie wurde in ein Krankenhaus auf dem sicheren Gebiet der Autonomen Region Kurdistan gebracht und dort behandelt. Diese extrem traumatische 
Erfahrung versetzte Ebru in einen Zustand der Handlungs- und Hilflosigkeit, welcher sie monatelang begleitete. Nachdem sie durch einen Freund über das Schicksal ihrer Herkunftsfamilie informiert worden war („Ich war sehr durcheinander in dieser Zeit, es war für mich wie ein Weltuntergang, weil ich genau wusste, was der IS mit Frauen macht"), beging Ebru einen Selbstmordversuch, welcher jedoch scheiterte. In diesem Zustand lebte Ebru weitere sechs Monate in einem Camp für yezidische Geflüchtete. Im Herbst 2015 entschied sie sich jedoch, weil sie für sich keine Zukunft mehr im Irak sah, für eine Flucht in Richtung Europa. Dabei erhielt sie zum einen Unterstützung durch Organisator*innen des Flüchtlingscamps, die die Flucht gemeinsam mit einer kurdischen Familie arrangierten. Zum anderen konnte Ebru anscheinend auf finanzielle Unterstützung von Freund*innen ihrer Familie zurückgreifen, ohne die eine Flucht wohl kaum zustande gekommen wäre.

Ebru flüchtete über die „Balkanroute“ (vgl. Hess et al. 2017) in Richtung Deutschland und versuchte dabei möglichst mit Menschen zu reisen, die yezidischer oder kurdischer Zugehörigkeit waren. Zudem vermied sie den Kontakt zu arabischen und/oder muslimischen (ausgenommen kurdischen) Menschen, da sie mit diesen eine Gefahr und Angst vor einer erneuten Verfolgung verband.

Verschiedene Grenzregime und die Migrationspolitiken verschiedener Länder erschwerten das Erreichen eines sicheren Ortes. So musste sich Ebru u.a. an der griechischen Grenze nach mehreren gescheiterten Überquerungsversuchen als Syrerin ausgeben, um über die Grenze zu gelangen, da die Einreise von Iraker*innen zu diesem Zeitpunkt (Winter 2015) vom griechischen Grenzschutz unterbunden wurde. In Griechenland entging sie nur durch eine medizinische Bescheinigung, die ihr ermöglichte, ihre körperlichen Verletzungen behandeln zu lassen, einer „Rückführung" in die Türkei. In Saloniki bezahlte Ebru einem Taxifahrer 50 Euro, um bis nach Mazedonien zu gelangen. Von dort reiste sie nach Österreich. Während Ebrus körperliche Verletzungen dazu beitrugen, dass ihre Schutzbedürftigkeit in den meisten Fällen anerkannt wurde, nutzte sie ihre ökonomischen Ressourcen, um Grenzen sowie längere Strecken nicht zu Fuß überwinden zu müssen.

Im Frühjahr 2016 erreichte Ebru im Alter von 20 Jahren Deutschland und befand sich folglich in der Situation, die Flucht zwar gemeistert zu haben, nun jedoch für die Etablierung eines sicheren Ortes ${ }^{5}$ sorgen zu müssen. Ebrus Hoffnungen, die sie in eine Verortung und in die Suche nach kurdischen und yezidischen Bezugspersonen in Deutschland setzte, wurden enttäuscht, wodurch sich Ebru in eine Situation der Handlungsunfähigkeit zurückgesetzt sah. Nachdem die Fluchtmigration eine tagtägliche Beschäftigung beinhaltet hatte, bei der sie sich um das Weiterkommen, die Übernachtung und die tägliche Verpflegung kümmern musste, gelangte Ebru, in Deutschland angekommen, in eine Situation, in der sie zum einen Zeit hatte,

\footnotetext{
${ }^{5}$ Mit der Suche nach einem sicheren Ort ist eine Lebensgrundlage gemeint, die Ebru eine Zukunftsperspektive in Deutschland sowie eine auf Dauer gestellte körperliche und seelische Unversehrtheit ermöglicht. In welcher Form sich dieser „,sichere Ort“ gestaltet, hängt dabei jedoch auch von dem Prozess des Ankommens in Deutschland ab, d.h. welche Möglichkeiten sich Ebru in Deutschland zur Selbstentfaltung und Teilhabe am gesellschaftlichen Leben bieten.
} 
über die Erlebnisse der Vergangenheit verstärkt nachzudenken. Zum anderen war sie - ganz ähnlich wie bei Arwa (s.u.) - für das Recht auf Asyl auf die Präsentation ihrer Verfolgungsgeschichte angewiesen, um die Anerkennung ihrer Schutzbedürftigkeit zu legitimieren. Auch im Ankommen fanden sich so rechtliche wie auch psychische Hürden, die den Ankunftsalltag Ebrus prägten. Über mehrere Stationen in verschiedenen Flüchtlingsunterkünften schaffte sie es schließlich, mit Unterstützung von Dolmetscher*innen, Freund*innen und Begleiter*innen, ein Jahr nach ihrer Ankunft in Deutschland in eine eigene Wohnung in einer hessischen Großstadt zu ziehen. Der Umzug bedeutete für Ebru die Etablierung eines sichereren Ortes, was sie selbst als Zugewinn von Handlungsmacht erlebte. Mittlerweile lebt auch eine von Ebrus Schwestern, die das Massaker von Kocho überlebt hat, in Deutschland. Im Rahmen des Asylverfahrens durchlebte Ebru einen gewissen Emanzipationsprozess, der sie in ihrer selbstbestimmten Entwicklung stärkte, die sich bereits in Ansätzen in ihrer Jugend bei der Loslösung von ihrer Herkunftsfamilie wiederfinden lässt. Zwar bleibt Ebru durch die kollektive Verfolgungsgeschichte mit den Yezid*innen verbunden, durch ihre Erlebnisse in der Vergangenheit sowie durch ihre neugewonnene Autonomie hat sie sich jedoch von einem Leben innerhalb einer yezidischen Community in Deutschland entfernt und distanziert sich so von ihrer Herkunftsgruppierung.

\section{Arwa, die Botschafterin, die für die Zukunft ihrer Familie kämpft}

Das mit Arwa (Jahrgang 1978) im Herbst 2016 auf Deutsch geführte Interview gibt Einsichten in einen Lebensverlauf einer yezidischen Frau aus dem Irak, die ihr Aufwachsen in einem Dorf nahe Mosul als eine Kontinuität der Verfolgung und als Fortbestehen eines Konfliktzustandes erlebte. Als sich Arwa und ihr Mann im Jahr 2009 entschieden, das Land aufgrund fehlender Zukunftsperspektiven für ihre Kinder zu verlassen, hatte(n) sie jahrelange Kriegszustände, Wirtschaftsembargos, den Dritten Golfkrieg sowie das Aufkommen militanter islamistischer Organisationen wie al-Qaida durchlebt. Das Interview mit Arwa wurde von Maria Fiona Fechter und Annette Andresen im Rahmen eines Lehrforschungsprojektes von Gabriele Rosenthal im August 2016 geführt und mir für meine Abschlussarbeit zur Verfügung gestellt. Der Kontakt zu Arwa entstand über eine Bildungs- und Beratungseinrichtung in Hannover, welche Deutschkurse - u.a. für Geflüchtete - anbot. Zum Zeitpunkt des Interviews lebte Arwa bereits seit sieben Jahren mit ihrer Familie in Deutschland. Bereits in einem ersten Vorgespräch erzählte sie von ihrer Herkunft und ihrer Familie, wobei sie auf ihre individuelle und kollektive Verfolgungsgeschichte hinwies, was im Interview noch stärker werden sollte. Die thematische Fokussierung auf die persönliche sowie kollektive Leidensgeschichte der Yezid*innen hat für Arwa auch die Funktion, Öffentlichkeit für deren Situation zu erzeugen. Da ein großer Teil ihrer Herkunftsfamilie weiterhin in einer prekären Situation im Irak 
lebt, ist dieses Präsentationsinteresse umso verständlicher. Zeitgleich erwartete Arwa zum Zeitpunkt des Interviews die Ankunft der Kinder ihrer Schwester aus dem Irak, die sie in ihre Obhut nehmen sollte und die wegen Komplikationen mit italienischen Behörden noch nicht in Deutschland angekommen waren. Von ihrer persönlichen sowie der kollektiven Leidensgeschichte der Yezid*innen zu berichten, bedeutete für sie, einen Umgang mit den Schuldgefühlen bezüglich der im Irak Zurückgebliebenen zu finden und gleichzeitig eine Legitimation für eine sichere Zukunft für Yezid*innen in und außerhalb Deutschlands zu schaffen. Die Analyse des biographischen Verlaufs von Arwa zeigt auf, dass dieser von einer Kontinuität der Verfolgungsgefahr im Irak geprägt ist. Dabei machte sie selbst früh in ihrem Leben Erfahrungen mit Verlusten innerhalb der Familie, die sich als Verfolgungserlebnisse einprägten und Arwas Zugehörigkeitsgefühl zur yezidischen Gruppierung stärkten. Arwas Bestreben, den eigenen Kindern eine sichere Zukunftsperspektive zu ermöglichen, lässt sich darüber hinaus als zentraler Antrieb für die Flucht und die Etablierung einer Bleibeperspektive in Deutschland verstehen.

Geboren in einem kleinen Dorf 50 Kilometer nördlich von Mosul im Jahr 1978, wuchs Arwa in einfachen Verhältnissen mit drei Schwestern und fünf Brüdern auf. Arwas Eltern waren bäuerliche Selbstversorger*innen. Sie besaßen mehrere Tiere und bauten Obst und Gemüse an. Ihre Kindheit erlebte Arwa im isolierten Rahmen des Elternhauses, während der Irak gleichzeitig erst von der Machtergreifung durch Saddam Hussein geprägt wurde und im Folgenden über mehrere Jahre Krieg mit dem Iran führte (1980-1988). Da die Diskriminierung und Verfolgung von religiösen und ethnischen Minderheiten im Irak unter Saddam Hussein als politisches Macht- und Unterdrückungsmittel genutzt wurde (siehe Abschnitt 2), hatten auch Arwa und ihre Familie Angst, Opfer von Verfolgung und Diskriminierung zu werden. Besonders die Schulbesuche ihrer Brüder sowie die Aufenthalte ihres Vaters außerhalb des eher sicheren Heimatdorfes bedeuteten für Arwa eine Bedrohung. Im Alter von neun Jahren (1988) erlebte Arwa zudem, wie die Bienenvölker der Familie, die einen großen Teil der eigenen Subsistenzwirtschaft ausmachten, in Folge der Chemiewaffenanschläge durch das irakische Regime im Nordirak starben, womit auch Arwa erste reale Konsequenzen des Iran-Irak-Krieges erfuhr. Zu einem einschneidenden Erlebnis in Arwas Leben kam es im Jahr 1989, Arwa war zu diesem Zeitpunkt zehn Jahre alt, als ihre damals 16-jährige und älteste Schwester durch unbekannte arabische Menschen getötet wurde, als diese mit Arwas Vater in den bergigeren Norden reiste, um landwirtschaftliche Erzeugnisse zu verkaufen:

Meine älteste Schwester war ungefähr 16 Jahre als sie einmal mit meinem Papa und den Schafen in die Berge gegangen ist, dort kamen arabische Männer mit Waffen und haben meine Schwester getötet. Ich habe es nicht gesehen, aber meine Mutter hat es mir erzählt.

Der Verlust der ältesten Schwester bedeutete für Arwa, dass die zumeist potentiell wahrgenommene und tradierte Bedrohung durch die arabische Bevölkerung zur ständigen Angst wurde. Der Schutzraum des Dorfes und der Familie wurde noch 
wichtiger, während sie leidvoll erlebte, dass ihr Vater das Leben ihrer Schwester nicht hatte beschützen können.

1990 begann der Zweite Golfkrieg zwischen dem Irak und Kuwait, womit Arwa mit zwölf Jahren die Kontinuität eines Konfliktzustandes erlebte. Wie unter Yezid*innen zu dieser Zeit durchaus üblich, heiratete Arwa mit 15 Jahren und zog in das nahegelegene Dorf ihres Ehemannes. In den Folgejahren gründete sie eine eigene Familie und kümmerte sich um den Haushalt und die Erziehung der Kinder. Zwischen 1994 und 2006 - Arwa war mittlerweile 28 Jahre alt - bekam sie sechs Kinder. Das Leben im Irak wurde in dieser Zeit weiterhin von Konflikten geprägt. Arwa erlebte den Dritten Golfkrieg (2003) und die anschließende Besatzungszeit. Es kam immer wieder zu größeren Anschlägen, Verfolgungen sowie Verschleppungen der yezidischen Bevölkerung durch radikal-muslimische Gruppierungen. Die Kontinuität des Konfliktzustandes, der vor allem nach 2003 beinahe alle Teile der Bevölkerung betraf, spiegelte sich auch in Arwas Rückblick auf ihr Leben im Irak wider:

Im Irak wir hatten immer Krieg, jeden Tag. Es gab immer Probleme, sie werfen Bomben in die Schule und in die Städte. Jeder hatte Angst Kinder in die Schule zu schicken oder in die Stadt zu gehen. Wir haben ganze Leben bisschen schwer, wir haben nicht gesehen schöne Tage.

Arwa und ihr Ehemann erlebten mit, wie yezidische Familien in ihrem Umfeld nach und nach die Flucht ergriffen. Auch für sie wurde deutlich, dass eine Zukunftsperspektive (,Im Irak wir haben viele Probleme, unsere Kinder sollen nicht so leben“), vor allem auch für ihre Kinder, im Irak von Ungewissheiten und Unsicherheiten geprägt sein würde. Im Jahr 2009 begann Arwa - sie war zu diesem Zeitpunkt 31 Jahre alt - mit ihrer Familie die Flucht in Richtung Deutschland. Zeitgleich mit dem Abzug eines Großteils der amerikanischen Truppen im Irak im Juni 2009 begann erst die älteste Tochter ${ }^{6}$ Arwas in Begleitung ihres Onkels die Flucht nach Deutschland, anschließend flüchteten Arwa, ihr Ehemann sowie ihre anderen fünf Kinder aus dem Irak in die Türkei. Da Arwa entweder kaum geographische Kenntnisse besitzt oder ihren Fluchtweg nicht genauer preisgeben möchte, ist ihre Fluchtroute nur bedingt nachvollziehbar, was sich im Interview deutlich widerspiegelte. Während der Flucht verließ sich Arwa auf die Leitung ihres Mannes, der den Kontakt zu Schleppern aufnahm. Deutschland kannte Arwa zu diesem Zeitpunkt nur aus Erzählungen. Einen Großteil der Strecke bestritt die Familie in einem LKW, die restlichen Wege fuhren sie mit einem Boot oder liefen zu Fuß. Bereits während ihrer Zeit in der Türkei wurde Arwa bewusst, dass die Flucht mit Kindern eine besondere Herausforderung darstellte. Ausreichend Verpflegung für die Kinder zu beschaffen, bedeutete für Arwa und ihren Ehemann, teilweise selbst darauf verzichten zu müssen. Die

\footnotetext{
${ }^{6}$ Arwa berichtete, dass sie ihre älteste Tochter im Alter von 15 Jahren vor allen anderen Familienmitgliedern zur Flucht aus dem Irak drängte, da die Familie Angst hatte, dass sie Opfer einer Entführung durch „schlechte“ muslimische Nachbarn werden könnte. Mit dem Begriff „schlechte Nachbarn“ bezog sich Arwa während des Interviews immer wieder auf solche muslimische/arabische Iraker, welche den Yezid*innen feindlich gestimmt waren und damit eine Gefahr für ihre Familie darstellten.
} 
Sicherheit der Kinder zu gewährleisten und sich gleichzeitig den Regeln der Schlepper unterordnen zu müssen, stellte für Arwa eine sehr belastende Aufgabe dar.

In der Türkei gelangte Arwas Familie bis zur Mittelmeerküste, von wo aus sie mit einem Schlepper in einem Boot mit ca. 20 weiteren Geflüchteten über das Mittelmeer fuhren. Während der nächtlichen Überquerung des Mittelmeers in Richtung Griechenland fiel einer von Arwas Söhnen ins Wasser. Während die Schlepper auf dem Boot Arwa drängten weiterzufahren, kämpfte sie vehement für die Suche und Rettung ihres Sohnes, der nicht schwimmen konnte. Arwa setzte sich letztlich gegen die Schlepper durch und konnte ihren Sohn aus dem Wasser retten. Das Erleben dieser traumatischen Situation stärkte Arwas Schutzinstinkt gegenüber ihren Kindern, während der willensstarke Einsatz zur Rettung ihres Kindes einen Zugewinn an Handlungsmacht bedeutete.

Den restlichen Fluchtweg - wahrscheinlich über die Balkanroute - bis nach Deutschland bestritt die Familie in einer größeren Gruppe entweder zu Fuß oder in einem LKW, wobei der Kontakt zu Schleppern eine wichtige Rolle spielte. Nach ungefähr einem Monat kamen sie in Deutschland an. Arwa und ihre Familie wurden in einer Flüchtlingsunterkunft in Hannover untergebracht. Wegen der Möglichkeit, dort in einer eigenen Wohnung zu leben, zog die Familie nach einiger Zeit nach Oldenburg. Dort lebte Arwa mit ihrer Familie zwar in Sicherheit, jedoch auch isoliert, was durch die fehlenden Sprachkenntnisse noch verstärkt wurde. Gleichzeitig beobachtete Arwa aus der Ferne, wie sich die Situation der zurückgebliebenen Familie zunehmend verschlechterte. Der plötzliche Tod ihres Vaters im Irak im Jahr 2012 verstärkte Arwas Kampf mit der Verantwortung für ihre zurückgebliebenen Familienangehörigen. Mit dem Erstarken des Daesh im Irak und dem Genozid im August 2014 flüchtete ihre restliche Herkunftsfamilie in Notunterkünfte auf kurdischem Regierungsgebiet. Die unsicheren Verhältnisse im Irak bestärkten Arwa darin, dass eine Rückkehr in den Irak keine Option war. Arwa versuchte, durch finanzielle Unterstützung und politische Öffentlichkeit auf das Schicksal der Yezid*innen im Irak aufmerksam zu machen. Gleichzeitig konzentrierte sie sich stärker auf die Etablierung ihrer Familie in Deutschland. Sie selbst begann einen Deutschkurs und suchte Kita- und Schulplätze für ihre Kinder. Da Arwa der Zugang zu Bildung in ihrer Kindheit und Jugend verwehrt geblieben war, wurde dieser ein zentrales Familienziel, welcher für die langfristige Bleibe- und Zukunftsperspektive in Deutschland umgesetzt werden sollte. Arwas Familie erhielt 2016 eine unbefristete Aufenthaltserlaubnis, womit eine langfristige Bleibeperspektive gesichert war. Arwa kämpft weiterhin mit ihrer Verantwortung gegenüber ihrer Familie im Irak. Im August 2016 nahm sie die geflüchteten Kinder ihrer Schwester auf und versuchte somit, die Sicherheit und Zukunftsperspektiven weiterer Familienmitglieder auszubauen sowie Verantwortung zu übernehmen.

Mit der Notwendigkeit, eine langfristige Bleibeperspektive in Deutschland zu etablieren, nahm Arwa neue Freiheiten und Rechte als Frau wahr, welche ihr im Irak nicht gegeben waren, und emanzipierte sich gegenüber der traditionell-yezidischen Rollenvorstellung. Dabei entfernte sie sich jedoch nicht - anders als Ebru - von 
ihrer yezidischen Herkunft, sondern empfindet sogar eine Stärkung ihres Zugehörigkeitsgefühls und versucht, auf die Verfolgung der Yezid*innen im Irak und damit deren Schutzbedürftigkeit hinzuweisen und Öffentlichkeit zu schaffen.

\section{$5 \quad$ Fazit}

Im Rahmen der biographischen Fallrekonstruktionen zu Ebru und Arwa wurde ein Einblick in die Prozesse der Zugehörigkeitskonstruktionen, der Flucht und des Ankommens von geflüchteten Yezidinnen aus dem Nordirak gegeben. Dabei wurde deutlich, dass das Erleben dieser Prozesse durch die in der Vergangenheit gemachten Erfahrungen geprägt ist und divergierende Perspektiven auf Gegenwart und Zukunft hervorbringt. Dies wurde bei Ebru und Arwa besonders durch den Wandel der eigenen Zugehörigkeitskonstruktionen, das Erleben von Handlungsmacht/Handlungsohnmacht sowie den Prozess des Ankommens in Deutschland deutlich.

Die biographischen Verläufe der beiden vorgestellten Frauen scheinen sich zunächst stark zu ähneln. Beide wuchsen in einer yezidischen Großfamilie auf, sind Teil einer religiösen Minderheit, flüchteten jeweils vor der Verfolgung aus dem Nordirak nach Deutschland und kämpfen heute für die Anerkennung der yezidischen Schutzbedürftigkeit. Beim genaueren Vergleich zeigen sich jedoch auch deutliche Unterschiede.

Diese werden bereits im unterschiedlichen Erleben von und Umgang mit Situationen in der Kindheit und Jugend deutlich, lassen sich noch stärker bei der Betrachtung der Verläufe der Verfolgungserlebnisse, der Flucht und der Ankunft in Deutschland nachvollziehen.

Während Ebru den Typus der „Neustarterin“ repräsentiert, die sich von ihrer yezidischen Zugehörigkeit stärker entfernt, repräsentiert Arwa den Typus der „Botschafterin“, die eine Stärkung ihres Zugehörigkeitsgefühls empfindet und sich aus der Präsentation der yezidischen Schutzbedürftigkeit handlungsmächtiger erlebt.

Bedeutend für die sich im Lebensverlauf wandelnden Zugehörigkeitskonstruktionen von Arwa und Ebru sind bereits die Erfahrungen und Erlebnisse in der Kindheit und Jugend. Der Wunsch nach Autonomie und Selbstbestimmung, den Ebru gegen den Willen ihrer Eltern durchsetzen konnte, steht konträr zu Arwas eher traditionellem und passivem Sozialisationsverlauf, der durch eine arrangierte Ehe und die Einnahme der Rolle der Mutter und Hausfrau geprägt war. Arwa identifizierte sich seit ihrer Kindheit stark mit der eigenen yezidischen Zugehörigkeit. Die religiöse Zugehörigkeit in Ebrus Familienhaus nahm hingegen nicht den höchsten Stellenwert ein. Wichtig zu unterscheiden ist auch, dass Arwa das Leben im Irak als Kontinuität eines Konfliktzustandes wahrnahm, während Ebru in ihrer isolierten sowie geschützten Lebenswelt innerhalb der yezidischen Community zunächst kaum Verfolgungserfahrungen machte. 
Der Verlust der Eltern und Geschwister sowie der Gründungsfamilie, der mit starker Traumatisierung und schwerer körperlicher Verletzung verbunden war, stellte einen Bruch in Ebrus Leben dar. Ein Zustand, der sie in völlige Hilflosigkeit versetzte. Die Entscheidung, das eigene Leben doch fortführen zu wollen, und die Erkenntnis, dass ihr Leben nur durch ein Verlassen des Iraks fortgeführt werden kann, versetzte Ebru in eine aktive Handlungsposition. Die Anerkennung ihrer eigenen Schutzbedürftigkeit wurde somit ihr wichtigstes Ziel. Ebru war während ihrer Flucht immer wieder auf sich selbst gestellt und musste Entscheidungen und Strategien entwickeln, die ihr ein Ankommen in einem sicheren Kontext ermöglichten. Ihre yezidische Zugehörigkeit spielte nur eine Rolle, wenn diese zum eigenen Schutz und zur Fortsetzung des Fluchtweges beitragen konnte.

Arwa hingegen erlebte keinen gewaltsamen Bruch in ihrem Leben. Das Verlassen des Iraks resultierte bei ihr eher aus einem Schutzauftrag gegenüber den eigenen Kindern. Damit übernahm sie zwar Verantwortung für ihre Familie, war jedoch auf Ressourcen und die Leitung des Migrationsprojektes durch andere angewiesen, womit sie in einer eher passiven Handlungsposition verharrte, in welcher der Schutz der Familie die wichtigste Aufgabe war.

Die Ankunft in Deutschland bedeutete für Ebru eine erneute Auseinandersetzung mit den eigenen Verlusten. Die Präsentation der Flucht- und Verfolgungsgeschichte wurde zur Anerkennung der eigenen Schutzbedürftigkeit sowie zum Erhalt eines legalen Status in Deutschland notwendig. Obgleich die Präsentation der yezidischen Zugehörigkeit für Ebru einen Schlüssel zum Erhalt eines Aufenthaltstitels bedeutete, ist ihr Rückzug aus der yezidischen Community und die Distanzierung von der eigenen Zugehörigkeit ein selbstgewählter Schritt, um ein autonomes Leben führen zu können. Die Distanzierung bietet dem Typus der „Neustarterin“ darüber hinaus erst die Möglichkeit, ein eigenes Leben - in Abstand zu ihren traumatisierenden Erfahrungen, jeglichen familialen Rückhalt zu verlieren - fortführen zu können.

Während die Flucht für Arwa und ihre Familie als schwierige und teils traumatisierende Erfahrung erlebt wurde, bei der die Sicherheit - vor allem der eigenen Kinder - immer wieder in Gefahr war, zog sich Arwa, in Deutschland angekommen, in den privaten Schutzraum der Familie zurück. Erst mit der Notwendigkeit, eine langfristige Bleibeperspektive im Ankunftskontext etablieren zu müssen, da eine Rückkehr in den Irak unwahrscheinlich wurde, begann Arwa, stärker am gesellschaftlichen Leben teilzunehmen und sich für die Anerkennung der eigenen Schutzbedürftigkeit einzusetzen. Die Stärkung und Präsentation der eigenen Zugehörigkeitskonstruktion bietet dem Typus der „Botschafterin“ hierbei die Chance, eine langfristige Bleibeperspektive im Ankunftskontext zu etablieren und zugleich Verantwortung gegenüber der zurückgebliebenen Familie im Irak zu übernehmen. Die eigene Zugehörigkeit wird von diesem Typus dabei als Handlungsmacht für die weitere Lebensgestaltung verstanden.

In beiden Fällen wurden durch die verstärkte gesellschaftliche Partizipation im Ankunftskontext Emanzipationsprozesse ausgelöst, die den Handlungsspielraum erweiterten. Während Ebru sich stärker von der yezidischen Zugehörigkeit distan- 
zierte, verstand Arwa die Nähe zur eigenen Zugehörigkeit als Stärkung ihrer eigenen Handlungsmacht. Darüber hinaus haben Ebru und Arwa gemein, dass sie durch ihre Bemühungen einen langfristigen Aufenthaltstitel erhalten haben, sich eine Zukunft in Deutschland aufbauen konnten und sich eine Rückkehr in den Irak nicht mehr vorstellen können. Beide profitierten von einer deutschen Migrationspolitik, welche die Schutzbedürftigkeit von Yezid*innen anerkannte. Die politischen Entwicklungen seit Herbst 2018 zeigen jedoch, dass die Anerkennung dieser Schutzbedürftigkeit nicht mehr flächendeckend gewährleistet wird und Rückführungen von Yezid*innen in den Irak immer öfter vorkommen - der Irak wurde wieder als ,sicheres Herkunftsland" betitelt und damit der Weg für Abschiebungen freigemacht. Doch ist der Irak wirklich sicher für Yezid*innen? Während der IS aus den Großstädten vertrieben werden konnte, gibt es weiterhin untergetauchte IS-Anhänger und weitere feindlich gestimmte Gruppen, von denen Gefahr für die yezidische Zivilbevölkerung ausgeht. Ein Ende von Diskriminierungen und Verfolgungen und somit ein Leben ohne Verfolgungsgefahr ist weiterhin nicht in Sicht. Es leben immerhin noch fast 100.000 Yezid*innen auf kurdischem Gebiet in Flüchtlingslagern, ohne eine Zukunftsperspektive in ihrer Heimat zu haben. Sie haben Angst, bei einer Rückkehr verfolgt zu werden. Dass Frauen, welche nach Vergewaltigungen durch IS-Anhänger Kinder geboren haben, vom Obersten Geistlichen Rat der Yezid*innen zudem vor die Wahl gestellt werden, sich entweder von ihren „nicht-yezidischen“ Kindern oder von der yezidischen Gemeinschaft zu trennen, bedeutet für viele Geflüchtete eine neue Form der Ausgrenzung. Für sie bedeutet das, dass sie weder zurückkehren noch ins Ausland migrieren können. Die Marginalisierung betrifft aktuell somit nicht nur yezidische Frauen, die auf der Flucht sind oder in einem Ankunftsland leben, sondern ebenso jene, die noch im Irak leben.

\section{Literatur}

Acikyildiz, B. (2010): The Yezidis. The History of a Community, Culture and Religion. London: Tauris.

Bundesamt für Migration (2017): Das Bundesamt in Zahlen 2016. Asyl, Migration und Integration. Online:

https://www.bamf.de/SharedDocs/Anlagen/DE/Statistik/BundesamtinZahl en/bundesamt-in-zahlen-2016.pdf $<04.04 .2020>$.

Buchta, W. (2016): Terror vor Europas Toren. Der islamische Staat, Iraks Zerfall und Amerikas Ohnmacht. Bonn: Bundeszentrale für politische Bildung.

Dinnayi, M. (2004): Yeziden im Irak. Eine bedrohte Minderheit ohne Existenzrechte. In: Kreuter, M./Schmidinger, T. (Hrsg.): Irak. Von der Republik der Angst zur bürgerlichen Demokratie. Freiburg: ça ira-Verlag, 197204. 
Heine, H. (2019): Wie die Jesiden im Irak um ihre Zukunft kämpfen. Der Tagesspiegel, 6. März 2019. Online: https://www.tagesspiegel.de/themen/ reportage/genozid-durch-islamischen-staat-wie-die-jesiden-im-irak-um-ihrezukunft-kaempfen/24064648.html <17.07.2020>.

Hess, S./Kasparek, B./Kron, S./Rodatz, M./Schwertl, M./Sontowski, S. (Hrsg.): Der lange Sommer der Migration. Grenzregime III. Berlin/Hamburg: Assoziation A.

Ibrahim, F. (2011): Droht eine Zerstörung der ethnischen und religiösen Vielfalt im Irak. Online: http://www.bpb.de/apuz/33465/droht-eine-zerstoerung-derethnischen-und-religioesen-vielfalt-im-irak?p $=$ all $<07.09 .2019>$.

Jorgow, C. (2019): Yezidische Frauen aus dem Irak. Leben mit der kollektiven und individuellen Verfolgungsvergangenheit. Unv. MA-Thesis. Göttingen: GeorgAugust-Universität Göttingen.

Kartal, C. (2016): Deutsche Yeziden. Geschichte, Gegenwart, Prognosen. Marburg: Tectum Verlag.

Kizilhan, J. I. (2019): Fünf Jahre nach dem Völkermord an Yeziden. Online: https://www.gfbv.de/fileadmin/redaktion/Reporte_Memoranden/2019/Gfb V-Dokumentation_zur_Lage_yezidischer_Frauen.pdf <18.09.2019>.

Oehring, O. (2017): Christen und Jesiden im Irak. Aktuelle Lage und Perspektiven. Online: https://www.kas.de/c/document_library/get_file?uuid=d67cc72543e3-f54a-3fb4-a9372bc72ba5\&groupId=252038<13.12.2019>.

Pro Asyl (2019): Fünf Jahre nach dem Genozid an den Jesid*innen im Irak. Online: https://www.proasyl.de/news/fuenf-jahre-nach-dem-genozid-an-denjesidinnen-im-irak/ <20.01.2019>.

Rosenthal, G. (2004): Ethnisierung der Biographie und Traumatisierung. In: Ottersbach, M./Yildiz, E. (Hrsg.): Migration in der metropolitanen Gesellschaft. Zwischen Ethnisierung und globaler Neuorientierung. Münster: Lit-Verlag, 217-227.

Rosenthal, G. (2015): Interpretative Sozialforschung. Eine Einführung. 5. Auflage. Weinheim: Beltz Juventa.

Rosenthal, G./Bahl, E./Worm, A. (2017): Illegalisierte Migrationsverläufe aus biografietheoretischer und figurationssoziologischer Perspektive. Die Landgrenze zwischen Spanien und Marokko. In: Forum Qualitative Sozialforschung, 17(3), Art. 10. Online: www.qualitativeresearch.net/index.php/fqs/article/view/2686/4012<16.07.2020>.

Savucu, H. (2016): Yeziden in Deutschland. Eine Religionsgemeinschaft zwischen Tradition, Integration und Assimilation. Marburg: Tectum. 
Schirrmeister, B. (2019): Jesiden jetzt schutzlos. In: Taz, 2. August 2019, online: https://taz.de/Urteil-zu-Gruppenverfolgung/!5610744/ <17.07.2020>

Schulz, A. (2009): Die besonderen traditionellen Regeln der Partnerwahl der Yeziden und deren Auswirkungen auf die Integration. Berlin: epubli.

Schütze, F. (1983): Biographieforschung und narratives Interview. In: Neue Praxis, 3, 283-293.

Sökefeld, M. (2019): Nations Rebound. German Politics of Deporting Afghans. In: International Quarterly for Asian Studies, 50 (1/2), 91-118.

Tagay, S. (2018): Die Jesiden. Religion, Gesellschaft und Kultur. Online: http://www.bpb.de/gesellschaft/bildung/filmbildung/270902/die-jesiden $<02.06 .2019>$.

Tagay, S./Ortac, S (2016): Die Eziden und das Ezidentum. Geschichte und Gegenwart einer vom Untergang bedrohten Religion. Hamburg: Landeszentrale für politische Bildung. 